The University of San Francisco

USF Scholarship: a digital repository @ Gleeson Library |

Geschke Center

Business Analytics and Information Systems

School of Management

2015

\title{
Risk Decomposition for Fund Managers
}

Matthew Dixon

University of San Francisco, mfdixon@usfca.edu

Follow this and additional works at: http://repository.usfca.edu/at

Part of the Finance and Financial Management Commons, and the Management Information Systems Commons

\section{Recommended Citation}

Dixon, Matthew Francis, Risk Decomposition for Fund Managers (May 31, 2015). R/Finance, Chicago, 2015.

This Conference Proceeding is brought to you for free and open access by the School of Management at USF Scholarship: a digital repository @ Gleeson Library | Geschke Center. It has been accepted for inclusion in Business Analytics and Information Systems by an authorized administrator of USF Scholarship: a digital repository @ Gleeson Library | Geschke Center. For more information, please contact repository@usfca.edu. 


\title{
Risk Decomposition for Fund Managers
}

\author{
Matthew Dixon ${ }^{1}$ \\ ${ }^{1}$ HedgeFacts LLP, 8180 Corporate Park Drive, Suite 140, Cincinnati, OH 45242.
}

February 23, 2015

Keywords: Component Value-at-Risk, Delta-Gamma, Investment Management, R

\begin{abstract}
This paper describes a methodology extension for decomposing non-linear portfolio risk by fund manager which we refer to as "Manager Component Value-at-Risk". The approach is well suited to funds holding any asset class or instrument type including derivatives. This decomposition approach is additive and fully captures the correlations between instrument returns and thus is well suited for decomposing risk by manager. We provide an example from a representative CTA portfolio that demonstrates superiority of the decomposition approach over other common practices for risk decomposition. The core methodology is implemented in $\mathrm{R}$ and made available to readers.
\end{abstract}

\section{Introduction}

Investment management firms seek to not only measure the Value-of-Risk of their portfolios but also measure the contribution of a sub-manager's positions to this Value-at-Risk. Examples of such funds include (i) large multi-strategy funds that employ multiple traders; large asset management firms such as pension funds, family offices and endowments; (ii) multi-manager 40-act investment funds; (iii) proprietary trading firms and (iv) fund of funds who receive position transparency.

Such a decomposition should be additive across fund sub-managers and fully capture the correlations between instrument returns in the portfolio. An additional preference is that sub-managers rank the positions in their "sub-portfolio" by their net exposure to the most significant market risk factors across the portfolio. Thus a sub-manager can concentrate on the position's in a sub-portfolio that are most significant to the overall VaR rather than those that are netted or hedged across the portfolio.

Through a representative CTA portfolio we illustrate how other decomposition approaches, such as simply measuring the VaR on the sub-manager's positions or incremental VaR [4] based on excluding the sub-portfolio, may yield mis-leading results.

This paper is structured as followed. In the following section, we define the terminology and notation used throughout the paper by revisiting the formulation of parametric delta-gamma VaR. One of the main contributions of this paper in given in Section 2.1, which introduces the non-linear methodology extension for component VaR. The definition of manager component $\mathrm{VaR}$ is given in Section 3. Section 4 demonstrates the decomposition approach, highlighting not only the importance of the convexity correction to account for non-linearity in the portfolio loss function, but also the limitations of other sub-manager risk decomposition approaches. A source listing for the implementation of the manager component VaR is given in Section 5. Section 6 concludes.

\section{Non-linear VaR}

The starting point for the non-linear risk methodology is a portfolio whose value $P\left(t ; R_{1}, R_{2}, \ldots, R_{N}\right)$ is a non-linear function in $N$ correlated market risk factors $R_{i}(t)$ at time $t$. The details of how the risk factors are chosen and what they really represent are germane, but for now we simply define them as any liquid and market instrument with respect to which the marginal risk of the portfolio is non-zero and linear. In other words, risk factors mostly exhibit quoted daily closes which are not stale and the portfolio must exhibit a linear factor exposure to each of the market risk factors. Examples include the S\&P 500 index for an equity portfolio or crude oil futures in a commodity portfolio. More often that not, however, the instrument itself is treated as the risk factor provided that itâs sufficiently liquid and is not a derivative. When the instrument in the portfolio is a derivative, the risk factor is chosen to be the underlying. In this case, the derivative position is represented as a non-linear function of the market risk factor and the linear model for portfolio loss becomes too restrictive.

Introducing the delta-gamma approximation $[1,2,3,4,5]$ under which the portfolio incurs a change of value on the profit and loss (P\&L) account due to market movements of the form 


$$
d P=\sum_{i, j} \underbrace{\Delta_{i} d R_{i}}_{\text {delta }}+\underbrace{\frac{1}{2} d R_{i} \Gamma_{i j} d R_{j}}_{\text {gamma }},
$$

where the first and second derivatives in the portfolio value with respect to the $i^{\text {th }}$ risk factor are denoted $\Delta_{i}=\frac{\partial P}{\partial R_{i}}$ and $\Gamma_{i j}=\frac{\partial^{2} P}{\partial R_{i} \partial R_{j}}$. The change in value of the $i^{t h}$ risk factor over a chosen time period is denoted $d R_{i}$. For linear portfolios, comprised of linear instruments such as stocks, the second (gamma) term may be neglected. Exclusion of the gamma term when the portfolio loss $-d P$ is non-linear in the risk factors, such as when options are held, leads to convexity errors in the portfolio loss which grow with the size of the derivatives position and the duration of the risk horizon.

Under the parametric delta-gamma approximation the risk factor returns are assumed to be normal. However, the addition of the convexity term in the portfolio loss function renders the portfolio returns non-linear. For mild departures from normality, the VaR of $d P_{t}$ over a period $d t$ and at a confidence level of $c$ can be estimated from a polynomial expansion in the first four moments of the loss distribution about the moments of the standard normal distribution. This expansion is referred to as a Cornish-Fisher expansion (see pages 284, 298, 317 [5]; [3]) and takes the form

$$
\operatorname{VaR}_{c, d t}\left[d P_{t}\right]=-\left(\mu_{1}+\left(z+\frac{1}{6}\left(z^{2}-1\right) s+\frac{1}{24}\left(z^{3}-3 z\right)(k-3)-\frac{1}{36}\left(2 z^{3}-5 z\right) s^{2}\right) \sqrt{\mu_{2}}\right.
$$

where $z=\Phi^{-1}(1-c)$ is the inverse standard normal cumulative distribution function $\Phi(z)$ evaluated at $1-c$. $c$ is the confidence limit and is typically between $95 \%$ and $99 \%$ corresponding to the respective 1 in 20 up to the 1 in 100 chance of encountering a severe loss. $s$ denotes skewness and is expressed in terms of the moments distribution of $d P_{t}$ from equation $(1)$ as $s=\frac{\mu_{3}}{\mu_{2}^{3 / 2}}$. Similarly, $\kappa$ denotes kurtosis and is given by $\frac{\mu_{4}}{\mu_{2}^{2}}$. For convenience, the first four moments of the distribution of $d P_{t}$ are specified here:

$$
\begin{aligned}
& \mu_{1}:=\mathbb{E}\left[d P_{t}\right]=\frac{1}{2} \operatorname{tr}(\Gamma \Sigma) \\
& \mu_{2}:=\mathbb{E}\left[d P_{t}-\mu_{1}\right]^{2}=\Delta^{T} \Sigma \Delta+\frac{1}{2} \operatorname{tr}(\Gamma \Sigma)^{2} \\
& \mu_{3}:=\mathbb{E}\left[d P_{t}-\mu_{1}\right]^{3}=3 \Delta^{T} \Sigma \Gamma \Sigma \Delta+\operatorname{tr}(\Gamma \Sigma)^{3} \\
& \mu_{4}:=\mathbb{E}\left[d P_{t}-\mu_{1}\right]^{3}=12 \Delta^{T} \Sigma(\Gamma \Sigma)^{2} \Delta+3 \operatorname{tr}(\Gamma \Sigma)^{4}+3 \mu_{2}^{2} .
\end{aligned}
$$

\section{$2.1 \quad$ Non-linear Component VaR}

Linear Component VaR as defined by Jorion (see page 160 of [4]) linearly decomposes the delta-normal VaR into instrument components for the purpose of assessing the contribution of each instrument's risk to the overall portfolio risk. For the deltanormal parametric approach, this computation is readily given since the portfolio loss is expressed as a linear function of the instruments.

When derivative positions are held in the portfolio, no such formula for component VaR is given that includes the convexity adjustment term in the portfolio loss function, and accounts for the non-normal loss distribution resulting from the convexity adjustment term. In order to estimate the component VaR, the convexity term must be separated into additive components attributed to each instrument. The intuitive decomposition approach taken in this paper rests on the observation that the linear component VaR for instrument $i$ can be expressed in terms of it's contribution to the standard deviation of the portfolio loss

$$
\left(\sigma_{P}^{[i]}\right)^{2}=\frac{1}{2} \sum_{k \in K_{i}} w_{i}\left(\Delta_{k}\right)\left(\nabla_{\Delta} \sigma_{P}\right)_{k}
$$

where $\nabla_{\Delta} \sigma_{P}=2 \Delta^{T} \Sigma$ is the sensitivity of $\sigma_{P}$ to $\Delta, w_{i}\left(\Delta_{k}\right)$ is the exposure of instrument $i$ to risk factor $k$ (or equivalently the contribution of instrument i to $\Delta_{k}$ ), and $K_{i}$ is the set of $k$ indices corresponding to the non-zero terms of $\mathbf{w}_{i}(\Delta)$.

The same is true for the convexity term - the contribution of the $i^{\text {th }}$ instrument to the convexity component is the $i^{\text {th }}$ diagonal component of the matrix-matrix product $w_{i}(\Gamma) \nabla_{\Gamma} \sigma_{P}$

$$
\left(\sigma_{P}^{[i]}\right)^{2}=\frac{1}{2} \sum_{k \in K_{i}} w_{i}\left(\Delta_{k}\right)\left(\nabla_{\Delta} \sigma_{P}\right)_{k}+\left(w_{i}(\Gamma) \nabla_{\Gamma} \sigma_{P}\right)_{k k}
$$

where $w_{i}(\Gamma)$ is a matrix whose $(l, m)^{t h}$ elements stores the contribution of instrument $i$ to $\Gamma_{l, m}$ and $\nabla_{\Gamma} \sigma_{P}=\Sigma \Gamma \Sigma$ is the matrix of sensitivities to $\Gamma$, whose $(l, m)^{t h}$ element is just the sensitivity of $\sigma_{P}$ to $\Gamma_{l m}$.

Since we have introduced a non-linear loss function, the component VaR is then written interms of the Cornish-Fisher expansion:

$$
\operatorname{VaR}_{c, d t}^{[i]}\left[d P_{t}\right]=-\left[\mu_{1}^{[i]}+\left(z+\frac{1}{6}\left(z^{2}-1\right) s+\frac{1}{24}\left(z^{3}-3 z\right)(\kappa-3)-\frac{1}{36}\left(2 z^{3}-5 z\right) s^{2}\right)\left(\sigma_{P}^{[i]}\right)^{2} / \sigma_{P}\right],
$$


where the $i^{t h}$ instrument's contribution to the first moment of the portfolio loss distribution is

$$
\mu_{1}^{[i]}=\frac{1}{2} \sum_{k \in K_{i}}\left(w_{i}(\Gamma) \Sigma\right)_{k k} .
$$

\section{Manager Component VaR}

The exposition thus far has just considered the contribution of each instrument to the VaR of the portfolio. The extension to Manager Component VaR follows trivially by allocation of instruments to sub-portfolio $j$, represented by the set of instrument indexes $I_{j}$, so that

$$
\widehat{\operatorname{VaR}}_{c, d t}^{[j]}\left[d P_{t}\right]=-\left[\hat{\mu}_{1}^{[j]}+\left(z+\frac{1}{6}\left(z^{2}-1\right) s+\frac{1}{24}\left(z^{3}-3 z\right)(\kappa-3)-\frac{1}{36}\left(2 z^{3}-5 z\right) s^{2}\right)\left(\hat{\sigma}_{P}^{[j]}\right)^{2} / \sigma_{P}\right],
$$

where

$$
\hat{\mu}_{i}^{[j]}=\frac{1}{2} \sum_{i \in I_{j}} \mu_{1}^{[i]}
$$

and

$$
\left(\hat{\sigma}_{P}^{[j]}\right)^{2}=\frac{1}{2} \sum_{i \in I_{j}}\left(\sigma_{P}^{[i]}\right)^{2}
$$

A key observation here is that if an instrument is net neutral across the entire portfolio then $\sigma_{P}^{[i]} \neq 0$ and so the manager component VaR is better interpreted as a relative measure and not an absolute one, since netting and hedging effects across sub-portfolios are not strictly accounted for. In other words, the portfolio may be neutral in an instrument, yet the instrument in the sub-portfolio will contribute to the manager component VaR unless its position is neutral in the sub-portfolio.

To ensure that the sub-manager is able to track the overall effect of the instrument on the portfolio VaR, we rank the instruments in the sub-portfolio manager's portfolio that are associated with the most significant risk factors. In this way, the sub-manager can focus on the risk of the most significant positions in their sub-portfolio that contribute to the overall portfolio VaR. It is also worth noting in passing that even a perfectly delta hedged position will still contribute to the manager component VaR due to the gamma risk.

\section{Results}

The following results demonstrate the application of the non-linear component VaR methodology to a representative portfolio, typical of that held by a CTA. The positions in the derivatives have, however, been exaggerated to highlight the importance of the convexity term. The allocation to sub-manager, here, is arbitrary made for illustrative purposes. Tables 1-3 show the holdings of each of three sub-portfolios, each owned by a sub-manager.

\begin{tabular}{|c|c|c|c|c|c|}
\hline Symbol & Expiry & Description & Sector & Holding & Currency \\
\hline EC & Sep 2014 & EURO FX CURR & Currencies & -27 & USD \\
BP & Sep 2014 & BRITISH POUND & Currencies & -52 & USD \\
HO & Sep 2014 & HEATING OIL & Energies & -10 & USD \\
CL & Sep 2014 & CRUDE OIL & Energies & -10 & USD \\
NG & Sep 2014 & HENRY HUB NATURAL GAS & Energies & -10 & USD \\
G & Sep 2014 & LONG GILT & Interest Rates & 15 & GBP \\
FGBL & Sep 2014 & Euro-Bund Futures & Interest Rates & 14 & EUR \\
\hline
\end{tabular}

Table 1: This table lists the holdings in sub-portfolio 1.

Table 4 compares the day-ahead delta Component VaR and delta-gamma Component VaR estimates for each instrument in the sub-portfolios at the $99 \%$ confidence level. The rank column ranks the importance of the associated risk factor to the overall portfolio VaR. Each symbol appears in descending order of rank. Thus the manager can discern their positions that are most critical to the overall risk of the portfolio as opposed to those positions that net out or are hedged across the entire portfolio.

We observe that when there is a strong component of non-linearity in the VaR, there are significant differences between the two estimates. This holds even when the instruments are linear because the portfolio loss distribution is non-linear under the delta-gamma portfolio loss function.

Table 5 shows the top ten risk factors ranked by their contribution to the overall delta-gamma VaR of the portfolio. 


\begin{tabular}{|c|c|c|c|c|c|}
\hline Symbol & Expiry & Description & Sector & Holding & Currency \\
\hline TY & Sep 2014 & 10 Year U.S. Treasury Notes & Interest Rates & -26 & USD \\
LH & Oct 2014 & LEAN HOGS & Livestock & 10 & USD \\
GC & Aug 2014 & GOLD & Metals & -7 & USD \\
VG & Sep 2014 & DJ EURO STOXX 50 & Stock Indices & -30 & EUR \\
ES & Sep 2014 & S\&P500 EMINI & Stock Indices & 50 & USD \\
L & Mar 2015 & 90DAY STERLING & Interest Rates & 45 & GBP \\
ED & Mar 2015 & EURODOLLAR & Interest Rates & -16 & USD \\
\hline
\end{tabular}

Table 2: This table lists the holdings in sub-portfolio 2.

\begin{tabular}{|c|c|c|c|c|c|}
\hline Symbol & Expiry & Description & Sector & Holding & Currency \\
\hline PUT NG 2.5 & Oct 2014 & HENRY HUB NATURAL GAS & Energies & 215 & USD \\
PUT NG 2.25 & Sep 2014 & HENRY HUB NATURAL GAS & Energies & 1600 & USD \\
PUT ES 1270 & Sep 2014 & S\&P500 EMINI & Stock Indices & -170 & USD \\
PUT CL 85 & Sep 2014 & CRUDE OIL & Energies & 82 & USD \\
CALL GC 1740 & Aug 2014 & GOLD & Metals & 2000 & USD \\
PUT NQ 2450 & Aug 2014 & NASDAQ 100 EMINI & Stock Indices & -196 & USD \\
\hline
\end{tabular}

Table 3: This table lists the holdings in sub-portfolio 3.

\begin{tabular}{|c|c|c|c|c|}
\hline Symbol & Expiry & 99\% Delta-Gamma Component VaR & 99\% Delta Component VaR & Risk Factor Rank \\
\hline $\mathrm{NG}$ & Sep 2014 & $\$ 13,519.09$ & $\$ 15,849.46$ & 1 \\
\hline CL & Sep 2014 & $\$ 15,762.75$ & $\$ 18,883.80$ & 2 \\
\hline $\mathrm{HO}$ & Sep 2014 & $\$ 14,737.84$ & $\$ 17,662.77$ & 4 \\
\hline $\mathrm{BP}$ & Sep 2014 & $\$ 12,617.50$ & $\$ 15,002.18$ & 5 \\
\hline $\mathrm{EC}$ & Sep 2014 & $\$ 11,766.81$ & $\$ 14,455.78$ & 6 \\
\hline $\mathrm{G}$ & Sep 2014 & $\$ 3,764.95$ & $\$ 4,659.97$ & 8 \\
\hline FGBL & Sep 2014 & $\$ 3,661.06$ & $\$ 4,549.75$ & 9 \\
\hline VG & Sep 2014 & $\$ 5,627.52$ & $\$ 6,753.13$ & 7 \\
\hline ED & Mar 2015 & $\$ 107.96$ & $\$ 106.10$ & 11 \\
\hline $\mathrm{L}$ & Mar 2015 & $\$ 10.34$ & $\$ 73.86$ & 12 \\
\hline LH & Oct 2014 & $-\$ 476.19$ & $-\$ 608.78$ & 13 \\
\hline TY & Sep 2014 & $-\$ 2,055.80$ & $-\$ 2,665.35$ & 14 \\
\hline ES & Sep 2014 & $-\$ 3,545.11$ & $-\$ 4,181.75$ & 15 \\
\hline GC & Aug 2014 & $\$ 4,691.23$ & $\$ 5,621.43$ & 16 \\
\hline PUT NG 2.25 & Sep 2014 & $\$ 88,568.48$ & $\$ 108,096.15$ & 1 \\
\hline PUT CL 85 & Sep 2014 & $\$ 35,052.87$ & $\$ 43,922.64$ & 2 \\
\hline PUT NG 2.5 & Oct 2014 & $\$ 38,985.18$ & $\$ 46,780.38$ & 3 \\
\hline PUT NQ 2450 & Aug 2014 & $\$ 1,281.51$ & $-\$ 66.31$ & 10 \\
\hline PUT ES 1270 & Sep 2014 & $-\$ 704.78$ & $-\$ 1,780.56$ & 15 \\
\hline CALL GC 1740 & Aug 2014 & $-\$ 8,979.55$ & $-\$ 6,897.13$ & 16 \\
\hline Sum & & $\$ 234,393.65$ & $\$ 286,217.53$ & \\
\hline 99\% Portfolio VaR & & $\$ 234,393.65$ & $\$ 286,217.53$ & \\
\hline
\end{tabular}

Table 4: This table compares the component VaRs for each instrument using the Delta and Delta-Gamma methodology. Each symbol is ranked by the contribution of it's associated risk factor to the overall portfolio VaR. 


\begin{tabular}{|c|c|c|}
\hline Risk Factor & Expiry & $99 \%$ Delta-Gamma Risk Factor Component VaR \\
\hline NG & Sep 2014 & $\$ 102,087.57$ \\
CL & Sep 2014 & $\$ 50,815.62$ \\
NG & Oct 2014 & $\$ 38,985.18$ \\
HO & Sep 2014 & $\$ 14,737.84$ \\
BP & Sep 2014 & $\$ 12,617.50$ \\
EC & Sep 2014 & $\$ 11,766.81$ \\
VG & Sep 2014 & $\$ 5,627.52$ \\
G & Sep 2014 & $\$ 3,764.95$ \\
FGBL & Sep 2014 & $\$ 3,661.06$ \\
NQ & Aug 2014 & $\$ 1,281.51$ \\
\hline
\end{tabular}

Table 5: This table show the top ten risk factors ranked by their contribution to the delta-gamma VaR of the portfolio. 
The comparative results of the three different manager risk decomposition approaches are shown in Table 6. Since the portfolio is composed of sub-portfolios, one approach taken by fund managers is to simply measure the VaR of each sub-portfolio. Clearly this risk measure is not additive across sub-portfolios since it is non-linear. It further suffers from ignoring the effect of correlation between the returns of instruments not in the sub-portfolio. For clarity of exposition, we have dubbed this approach "Independent VaR".

Recall that the incremental VaR measures the difference between the VaR of the portfolio and the VaR of the portfolio without the sub-portfolio. Table 6 shows that removing sub-portfolio 3 has the largest effect on the VaR. We also observe that the VaR excluding sub-portfolio 2, is higher than the portfolio VaR, suggesting that sub-portfolio 2 has an exposure reducing effect. We note, however, that incremental VaR is not additive and it also does not fully capture the effect of correlations between the returns of instruments in the sub-portfolio and the returns of those instruments in the remainder in the portfolio but not in the sub-portfolio. Hence it is difficult to reliably interpret these results.

The Manager Component VaR reveals a different view on the sub-manager's contribution to risk than the other two methods. The Component VaR shows that independent VaR and incremental VaR over and underestimate the contribution of all subportfolios to the overall risk respectively. These discrepancies can be explained by the fact that component VaR is fully capturing the effect of correlations between all instrument returns. We further confirm numerically that our derivation of the non-linear component $\mathrm{VaR}$ is an additive measure and thus the sum of components equals the delta-gamma VaR estimate given in Table 4.

\begin{tabular}{|c|c|c|c|c|}
\hline VaR Methodology & Sub-manager 1 & Sub-manager 2 & Sub-manager 3 & Sum \\
\hline Independent & $\$ 176,615.25$ & $\$ 78,558.35$ & $\$ 179,596.88$ & $\$ 434,770.48$ \\
Incremental & $\$ 15,486.59$ & $-\$ 823.96$ & $\$ 75,584.06$ & $\$ 90,246.69$ \\
Component & $\$ 75,830.01$ & $\$ 4,359.95$ & $\$ 154,203.70$ & $\$ 234,393.65$ \\
\hline
\end{tabular}

Table 6: This table compares three common approaches for risk decomposition across sub-managers, each using delta-gamma VaR. We confirm numerically that only the Component VaR is additive.

\section{$5 \quad$ R Code}

The following source listing shows the primary calculation of the manager component VaR. In the listing, the Delta, Gamma and Sigma matrices are assumed to have already been calculated. The implementation assumes that S4 classes are defined for portfolio, manager and instrument. CalcMoments is a global function that calculates the moments of the portfolio loss distribution. calcComponentSigma and calcComponentMu are member functions of the instrument class that computes the component variance and mean respectively. The definition of the classes and functions are available to the reader on request.

Listing 1: Source listing for demonstration of the manager component VaR calculation.

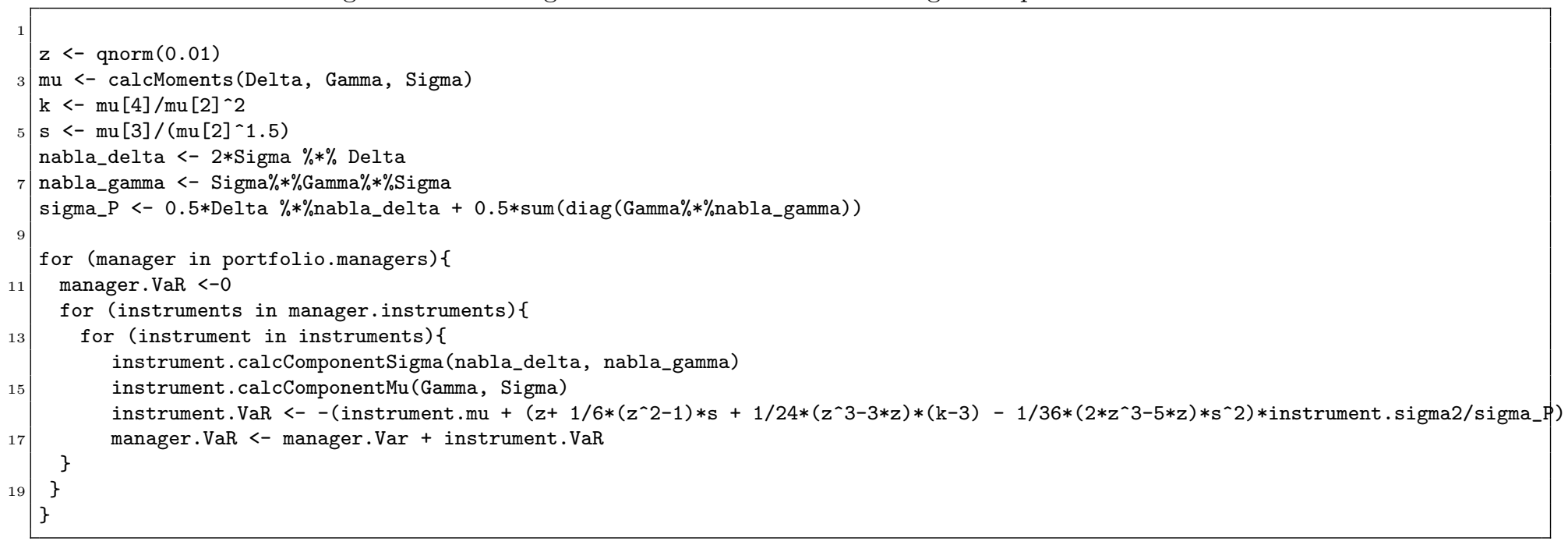

\section{Conclusion}

This paper describes a methodology extension for decomposing non-linear portfolio risk by fund manager which we refer to as "Manager Component Value-at-Risk". The approach is well suited to funds holding any asset class or instrument type including derivatives. This decomposition approach is additive and fully captures the correlations between instrument returns and thus is well suited for decomposing risk by manager. We provide an example from a representative CTA portfolio that demonstrates 
superiority of the decomposition approach over other common practices for risk decomposition. The core methodology is implemented in $\mathrm{R}$ and is available to readers on request.

\section{References}

[1] Alexander C.,Market Risk Analysis: Value-at-Risk, part IV, 2008.

[2] Britten-Jones M. and Schaefer S.M., Non-Linear Value-at-Risk, European Finance Review, 2004.

[3] Castellacci F. and Siclari M.J., The Practice of Delta-Gamma VaR: Implementing the Quadratic Portfolio Model, European Journal of Operational Research, 2003, pp. 529-545.

[4] Jorion P., Value-at-Risk, Second Edition.

[5] Meucci A., Risk and Asset Allocation, Springer Finance, 2005. 